\title{
Comprehensive analysis of bZIP transcription factors uncovers their roles during dimorphic floret differentiation and stress response in Cleistogenes songorica
}

\author{
Qi Yan, Fan Wu, Tiantian Ma, Xifang Zong, Qian Ma, Jie Li, Yufeng Zhao, Yanrong Wang and Jiyu Zhang* (D)
}

\begin{abstract}
Background: Transcription factors act as important regulators of transcription networks. Basic leucine zipper (bZIP) transcription factors have been shown to be involved in multiple biological processes in plants. However, no information is available for the bZIP family in Cleistogenes songorica, which is an important xerophytic and allotetraploid grass in desert grasslands.

Results: In this study, 86 CsbZIPs were identified in the allotetraploid C. songorica genome. For location analysis, CsbZIPs were distributed evenly across two subgenomes of $C$. songorica. Phylogenetic tree analysis among three species indicated that CsbZIPs were evolutionarily more closely related to OsbZIPs than AtbZIPs. Syntenic and phylogenetic analyses confirmed that the CsbZIPs were mainly expanded by whole-genome duplication events. Furthermore, it was determined that rice and C. songorica might have undergone purified selection during their long evolutionary history by calculating the Ks values and $\mathrm{Ka} / \mathrm{Ks}$ ratios of orthologous gene pairs. By analysing the expression patterns of CsbZIPs in different tissues and under abiotic stresses, 21 CsbZIP genes were differentially expressed between chasmogamous $(\mathrm{CH})$ and cleistogamous (CL) flowers, including two FLOWERING LOCUS D (FD) genes. In shoots and roots, 79.1 and $87.2 \%$ of the CsbZIP genes, respectively, displayed transcript changes under at least one stress treatment, such as heat, cold, drought and salt. Strikingly, 17 common CsbZIP genes showed differential expression under stress response and during $\mathrm{CL}$ flowering. Co-expression network, GO annotation and real-time quantitative reverse transcription PCR (qRT-PCR) analyses revealed a close relationship between $C L$ flowering-associated genes and abiotic stress-related genes.
\end{abstract}

Conclusions: BZIP TFs were comprehensively analysed and identified in allotetraploid C. songorica. Our results provide insights into the evolutionary history of the bZIP family in C. songorica and provide abiotic stress-responsive and CL-associated candidate CsbZIP genes for potential applications in the genetic improvement of plants.

Keywords: Cleistogenes songorica, Transcription factor, BZIP genes, Evolutionary analysis, Cleistogamous, Stress response

\section{Background}

Transcription factors (TFs) regulate the expression of functional genes by interacting with downstream promoter regions. The bZIP TF family is one of the largest and most conserved TF families in plants [1]. In general, the bZIP domain contains a conserved basic region and

\footnotetext{
* Correspondence: zhangjy@|zu.edu.cn

State Key Laboratory of Grassland Agro-ecosystems, Key Laboratory of Grassland Livestock Industry Innovation, Ministry of Agriculture and Rural Affairs, College of Pastoral Agriculture Science and Technology, Lanzhou University, Lanzhou 730020, People's Republic of China
}

a leucine zipper region $[2,3]$. The basic region is composed of a conserved $\mathrm{N}-\mathrm{x}_{7}-\mathrm{R} / \mathrm{K}$ motif within 18 amino acid residues $[4,5]$. The leucine zipper region contains several leucine repeats or hydrophobic amino acids [6]. The basic region and leucine zipper region are responsible for DNA binding and specific DNA identification, respectively. Furthermore, bZIP TFs can be divided into several subfamilies based on their conserved motifs. For example, the subfamily A bZIP TFs contain abscisic acid (ABA)-responsive elements in the promotor region,

(c) The Author(s). 2019 Open Access This article is distributed under the terms of the Creative Commons Attribution 4.0 International License (http://creativecommons.org/licenses/by/4.0/), which permits unrestricted use, distribution, and reproduction in any medium, provided you give appropriate credit to the original author(s) and the source, provide a link to the Creative Commons license, and indicate if changes were made. The Creative Commons Public Domain Dedication waiver (http://creativecommons.org/publicdomain/zero/1.0/) applies to the data made available in this article, unless otherwise stated. 
named ABA-responsive element binding proteins (AREB) or AREB binding factors (ABF), which have been identified to play an important role in stress signalling [7, 8]. The subfamily S bZIP TFs respond to extreme environmental stress [9].

BZIP TFs have been demonstrated to be involved in many important biological processes in plants, such as flowering, lateral root development, biomass, lipid productivity, pollen germination and seed maturation [10-14]. For example, FLOWERING LOCUS D (FD), which encodes a bZIP transcription factor, is mainly expressed in the shoot apex and is required for FLOWERING LOCUS $T(F T)$ to initiate flowering in Arabidopsis thaliana [10]. In Arabidopsis, $f d$ mutants showed a late flowering phenotype [10]. AtFD overexpression caused a reduction in plant height and spikelet size in transgenic rice plants [15]. In addition, AtbZIP34 has been characterized as involved in pollen wall patterning by controlling many metabolic pathways of lipid metabolism and/or cellular transport in Arabidopsis [16]. The lipid content and biomass of transgenic Nannochloropsis salina was increased by overexpressing NsbZIP1 [11]. Increasing evidence has indicated that bZIP TFs play a central role in the regulation of plant responses to biotic and abiotic stress, including water deficit [17], salt toxicity [18], temperature fluctuations [19], abscisic acid (ABA), gibberellic acid signalling [20], methyl jasmonate signalling [21] and defence against pathogens [22, 23]. For instance, overexpression of MebZIP3 and MebZIP5 improved the disease resistance for cassava bacterial blight in tobacco [24]. In tomato, the transcription factor SlbZIP1 is involved in salt and drought resistance by regulating ABA biosynthesis [25]. The OsbZIP71 RNAi knockdown transgenic plants were highly sensitive to salt and polyethylene glycol stress [26]. Together, these studies showed that bZIPs are widely involved in biological processes and various stresses in plants.

Currently, bZIP TFs have been identified in numerous species, such as Arabidopsis [9], Oryza sativa [1], Brassica napus [27], strawberry [28], Brachypodium distachyon and cassava with genome sequencing $[4,5]$. However, no studies have characterized and identified bZIP family members in C. songorica, which is one of the most important native perennial forage and ecological grasses in the desert grassland of Northwest China. C. songorica, an allotetraploid plant, can grow in semi-arid and desert areas where the mean annual rainfall is $110 \mathrm{~mm}$ [29]. Strikingly, C. songorica can produce both chasmogamous $(\mathrm{CH})$ and cleistogamous (CL) flowers on a plant, but they appear in different locations [30]. Additionally, these two types of flowers have some differences in the morphology of their floral organs, such as lodicule size, pollen, lemma and stigma length. In Arabidopsis and alfalfa, overexpression of the C. songorica LEA and CsALDH genes improved the tolerance to drought and salt stress in transgenic lines [31-34]. Previously, we obtained a high-quality genome sequence and expression data under abiotic conditions for $C$. songorica (data not published). Understanding the molecular mechanisms by which the bZIP genes of $C$. songorica respond to abiotic stress and CL flowering may provide a valuable genetic resource for the improvement of other grasses and crops. In this study, the bZIP family was identified in the $C$. songorica genome to reveal the phylogenetic relationships, conserved motifs, gene structures, synteny, co-expression networks, and cis-elements of the bZIP TFs. In addition, the CL flowering and abiotic stress-related genes were also analysed.

\section{Results}

\section{Genome-wide identification of bZIP family genes in $C$. songorica}

In this study, 86 predicted $C s b Z I P$ genes were identified from the $C$. songorica genome, named CsbZIP1 to CsbZIP86 (Additional file 1: Table S1). Furthermore, the amino acid residues, grand average of hydropathicity (GRAVY), isoelectric points (pIs), molecular weights (Mws), and CDS lengths of the CsbZIP proteins were analysed. The 86 CsbZIP proteins varied from 122 (CsbZIP68) to 1032 (CsbZIP79) amino acid residues, the CDSs were distributed from 377 to $3175 \mathrm{bp}$, the relative Mws ranged from 13.8 (CsbZIP19) to 113.4 (CsbZIP79) $\mathrm{KDa}$, the GRAVY values ranged from - 1.194 (CsbZIP30) to 0.483 (CsbZIP69), and the pIs ranged from 4.81 (CsbZIP68) to 11.7 (CsbZIP47; Additional file 1: Table S1). The analysis of subcellular location showed that 92 (95.3\%) CsbZIP proteins were anchored in the nucleus. In subfamily B, two and one CsbZIP proteins (60\%) were anchored in the endoplasmic reticulum and plasma membrane, respectively. In addition, CsbZIP70 were anchored in chloroplast (Additional file 1: Table S1).

To determine the evolutionary relationship of the CsbZIP proteins and other known bZIP proteins, an unrooted neighbour-joining tree was created with 250 bZIP proteins from three plant species (86 from $C$. songorica, 75 from Arabidopsis, and 89 from rice). The results showed that 250 bZIP proteins were grouped into 10 subfamilies, named subfamilies A to I and S. Subfamily A and subfamily S both contained 25 CsbZIP protein members, whereas no CsbZIP proteins were found in subfamily F (Fig. 1). Generally, the CsbZIP proteins had closer relationships with the bZIPs from rice than those from Arabidopsis, which is confirmed by the current understanding of plant evolutionary history. To further understand the structural evolution of the $C s b Z I P$ genes, we analysed the gene structure of the CsbZIP genes. Twenty-one CsbZIP genes (24.4\%) had one exon, 19 of which were clustered into subfamily A. CsbZIP79, CsbZIP85 and CsbZIP22 contained 11 


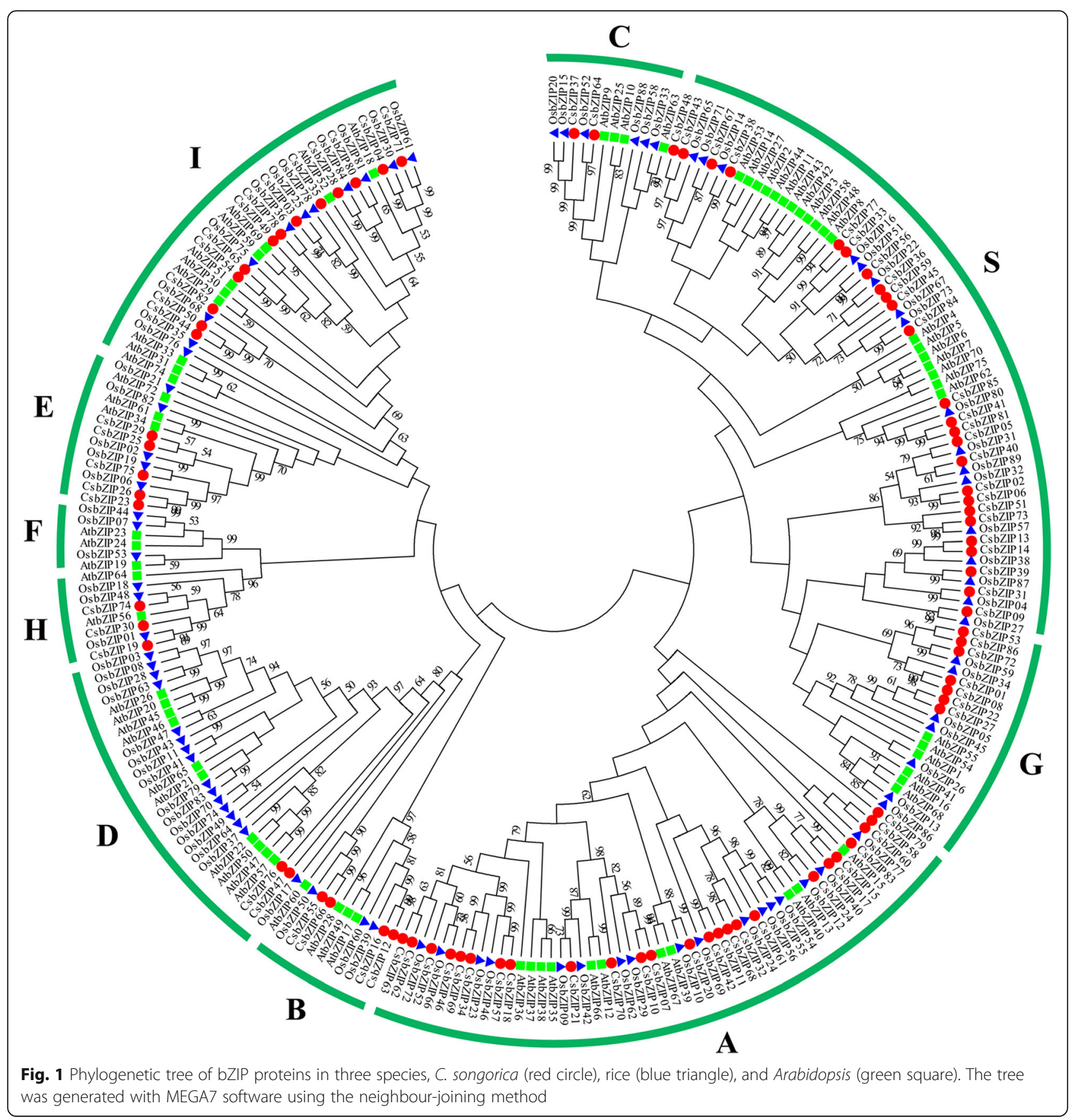

exons, which was the highest number of exons among the CsbZIP genes. In subfamilies $\mathrm{A}, \mathrm{B}, \mathrm{E}, \mathrm{H}$, and $\mathrm{I}$, most $C s b Z I P$ genes contained $<5$ exons, and most of the CsbZIP genes in subfamilies C, D, and G contained 6-11 exons (Additional file 2: Figure S1). To obtain insight into the diversity of motifs and functional prediction of the CsbZIP proteins, 20 conserved motifs were identified and designated using the MEME web server. Strikingly, all CsbZIP proteins contained the basic leucine zipper domain motif 1 . In addition, motifs 9,13 and 15 were specific for subfamily I, motif 20 was only found in subfamily
$\mathrm{H}$ and $\mathrm{B}$, motifs 3, 4 (abscisic acid-insensitive), 5 and 6 only appeared in subfamily A, and motif 12 was present exclusively in subfamily G (Additional file 2: Figure S1 and Additional file 3: Figure S2). In summary, the CsbZIP genes have conserved structural and exonintron organization similarities in the same subfamily.

\section{Genome synteny and variation analysis of the bZIP family in C. songorica}

For gene loci analysis, 82 CsbZIP genes (95.34\%) were located on 20 C. songorica chromosomes, with chromosome 
CsA11 containing the most (12.2\%), followed by chromosomes CsA04, CsB08 and CsA02 with approximately 8.5\%, but no CsbZIP genes were located on chromosome CsB19 (Additional file 4: Figure S3). Subgenome A and subgenome B of C. songorica contained 44 (53.7\%) and 38 (46.3\%) CsbZIP genes, respectively. The average amino acid number and pIs of the CsbZIP proteins in subgenome A were higher than those of the CsbZIP proteins in subgenome B (Additional file 4: Figure S3). In this study, 40 putative paralogous gene pairs were identified in the $C$. songorica genome, including 39 paralogous gene pairs produced by segmental duplication, and 1 paralogous gene pair produced by tandem duplication events with the same chromosomes (CsbZIP32 and CsbZIP68). In addition, 29 paralogous gene pairs were identified from subgenome A to subgenome B of $C$. songorica (Fig. 2 and Additional file 5: Table S2). For example, CsbZIP14 and CsbZIP15 were located on CsB13, CsbZIP13 and CsbZIP17 on CsA14, whose gene structure and conserved motif were highly similar. For further evolutionary studies of the bZIP family, we calculated the $\mathrm{Ka}, \mathrm{Ks}$ and $\mathrm{Ka} / \mathrm{Ks}$ values of paralogous gene pairs based on synteny analysis. For the paralogous gene pairs in C. songorica, the frequency distributions of the relative $\mathrm{Ks}$ for the paralogous gene pairs peaked at $0-0.4$ in C. songorica (Fig. 4 and Additional file 5: Table S2).

\section{Evolutionary and phylogenetic relationship of $C$. songorica and rice bZIP TFs}

We further performed a comparative bZIP synteny map of the rice and $C$. songorica genomes. Fifty-four rice $b Z I P$ genes and 72 CsbZIPs were identified as orthologous by large-scale syntenies (Fig. 3 and Additional file 6: Table S3). Among them, we found 12 pairs of syntenic orthologous genes (one-to-one), including CsbZIP79-OsbZIP60, CsbZIP78-OsbZIP36, CsbZIP67-OsbZIP71 and CsbZIP05OsbZIP31. The results suggested that these genes were derived from the same ancestor of rice and $C$. songorica. The relationship of one CsbZIP gene corresponding to multiple OsbZIP genes was also found, such as CsbZIP84OsbZIP67/OsbZIP73 and CsbZIP17-OsbZIP12/OsbZIP40. Additionally, syntenic orthologous gene pairs of one OsbZIP corresponded to multiple CsbZIP genes. For instance, OsbZIP77-CsbZIP58/CsbZIP60, OsbZIP78-CsbZIP03/ CsbZIP35, and OsbZIP72-CsbZIP46/CsbZIP52/CsbZIP62/ CsbZIP63. Strikingly, syntenic orthologous gene pairs with four CsbZIP genes corresponded to the same two genes: CsbZIP63/CsbZIP62/CsbZIP52/CsbZIP46-OsbZIP72/ OsbZIP66 (Fig. 3 and Additional file 6: Table S3). For the duplicated orthologous gene pairs, the Ks peaked at 0.4-1.6 between $C$. songorica and rice (Fig. 4 and Additional file 6: Table S3). The $\mathrm{Ka} / \mathrm{Ks}$ ratios peaked between $0.08-0.2$ for the paralogous gene pairs, and the $\mathrm{Ka} / \mathrm{Ks}$ ratios between the rice and C. songorica genomes were distributed at $0.08-0.24$, with the highest in the CsbZIP01-OsbZIP34 pair $(\mathrm{Ka} / \mathrm{Ks}=$ $0.58)$.

\section{Identification of cis-elements in the CsbZIP promoters and LTR retrotransposon insertions in the CsbZIP introns}

To explore the mechanisms of the CsbZIP genes in the stress response and developmental process, we searched for 18 cis-elements in the promoter region of the CsbZIP genes, which were predicted to be involved in development, abiotic stress and phytohormone response. The six most common cis-elements included the TGACG-motif (89.5\%), abscisic acid responsive element (ABRE, 89.5\%), CAT-box elements (53.4\%), drought stress element (MBS, 52.3\%) and LTR (lowtemperature responsiveness, $36 \%$ ) in the CsbZIP promoters (Fig. 5a and Additional file 6: Table S3). The CGTCA-motif and TGACG-motif are involved in MeJA stress, while ABREs are involved in ABA stress. The CAT-box elements and MBS are involved in development and drought stress, respectively. Additionally, 30 CsbZIP genes contained the salicylic acid responsive elements (TCA-element), which were involved in the salicylic acid response. Notably, the promoters of 13 CsbZIP genes (15.1\%) contained at least six cis-elements (Additional file 7: Table S4). The analysis of chromosome distributions indicated that different cis-elements preferred some chromosomes. For distance, TGAelements were enriched in CsB02 and CsA12. Likewise, some cis-elements were distributed in rare chromosomes, such as GCN4_motif (Fig. 5c and Additional file 6: Table S3). Subfamily G contained all identified cis-elements in the promoters of the CsbZIP genes. Compared with other families, subfamily $\mathrm{S}$ had the most LTRs, MBSs, ABREs and TC-repeats (Fig. 5b and Additional file 7: Table S4).

Insertion of the LTR retrotransposon may affect gene structure and gene expression. Here, 14 CsbZIP genes (16.3\%) were identified for the insertion of the LTR retrotransposon in the intron region (Fig. 6). CsbZIP63 contained the most LTR retrotransposon elements, including 5 copia elements, 4 gypsy elements and 1 other element. CsbZIP46, CsbZIP72, CsbZIP07 and CsbZIP37 only contained one gypsy element. In contrast, CsbZIP76 and CsbZIP71 only contained one copia element. Furthermore, 4 CsbZIP genes contained both copia and gypsy elements (Fig. 6).

\section{Identification of dimorphic floret-associated bZIP family members}

To study the expression pattern of the CsbZIP genes in different organs, we performed transcriptome analysis of C. songorica tissues, including the chasmogamous $(\mathrm{CH})$ flowers, cleistogamous (CL) flowers, seeds, leaves, and roots (Fig. 7a). Eighty-one (94.2\%) CsbZIP genes expressed at least in one tissue $(\mathrm{FPKM} \geq 1)$, and $40(46.5 \%)$ genes 


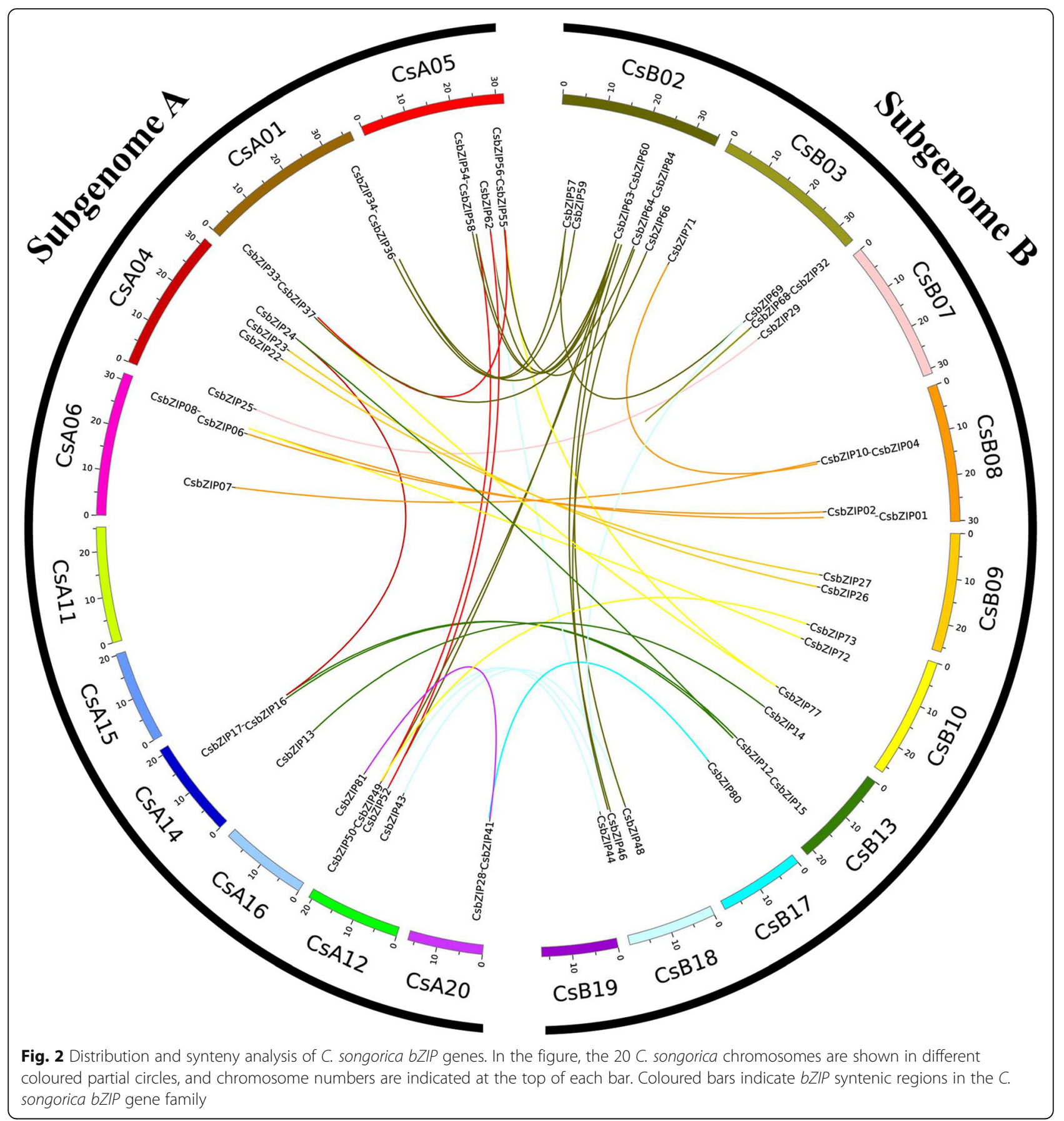

were expressed in all tissues (FPKM $\geq 1$; Additional file 8: Table S5). Furthermore, 33 genes showed high expression levels in at least one tissue (FPKM $\geq 20)$. Three genes were highly expressed in all tissues, including CsbZIP10, CsbZIP13 and CsbZIP38 (Fig. 7b). Strikingly, nine genes were only highly expressed in flowers but not in roots and leaves tissues; five genes showed high expression levels only in roots but not in flower tissues (Fig. 7b).

C. songorica can produce dimorphic floret (chasmogamous, cleistogamous) on the same individual plant that appear in different positions. We identified 21 differentially expressed $\mid \log _{2}$ (fold change) $\geq 1 \mid C s b Z I P$ genes between $\mathrm{CH}$ and $\mathrm{CL}$ flowers, including five downregulated and 16 upregulated CsbZIP (Fig. 7c). Gene ontology (GO) annotation showed that these genes were involved in the regulation of biological process (GO:0050789), developmental maturation (GO:0021700), gametophyte development (GO:0048229), response to stress (GO:0006950), pollen development and maturation (GO:0009555 and GO:0010152). Strikingly, CsbZIP58 and CsbZIP60 were 


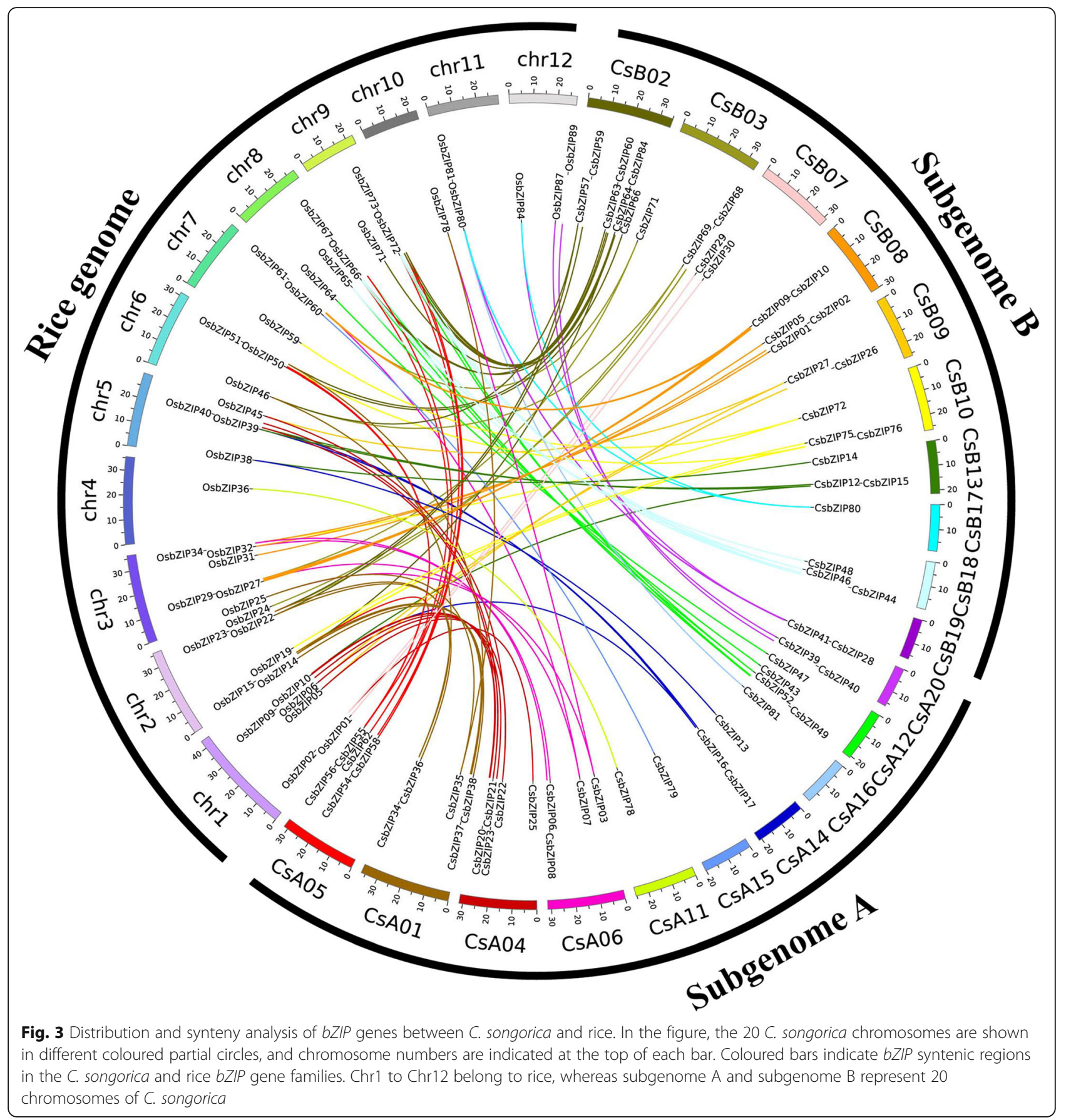

identified as FD (FLOWERING LOCUS D) gene, which participated in floral induction. CsbZIP60 participated in pollen maturation (GO:0010152) and pollen development (GO:0009555) (Additional file 9: Table S6).

Four dimorphic florets related CsbZIP genes (CsbZIP20, CsbZIP57, CsbZIP59 and CsbZIP82) were selected for coexpression analysis (Fig. $7 \mathrm{~d}$ and Additional file 10: Table S7). Nine hundred and sixty co-expressed genes were identified and some showed overlap with these four genes. Gene ontology analysis indicated that these genes were involved in organ development (GO:0048513), flower development (GO:0009908), floral organ development (GO:0048437), reproductive structure development (GO:0048608) and response to stress (GO:0006950; Additional file 10: Table S7 and Additional file 11: Figure S4). This result suggested that the CsbZIP genes may participate in dimorphic florets development by regulating floral organ development and flower development. 


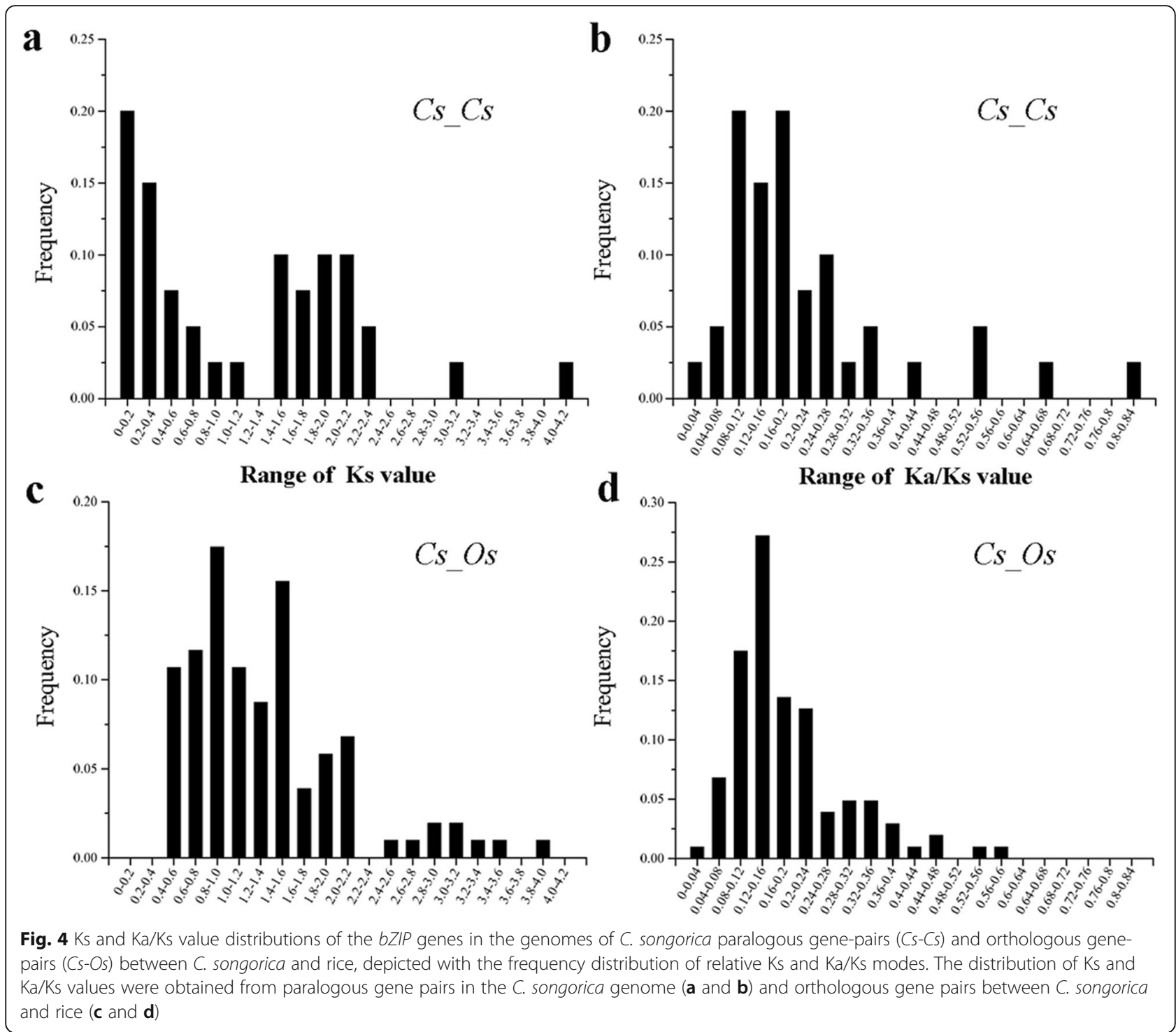

\section{Identification of abiotic stress-related CsbZIP genes}

We used the transcriptome data sets of $C$. songorica treated with high temperature (heat), low temperature (cold), salt and drought stress to determine the impact of the CsbZIP genes on abiotic stress. Seventy-four (87.2\%) and seventy-eight (90.7\%) CsbZIP genes showed gene expression at least in one stress condition (FPKM $\geq 1$ ) in shoots and roots (Fig. 8a and Additional file 12: Table S8), respectively. Furthermore, 68 (79.1\%) and 75 (87.2\%) CsbZIP genes were differentially expressed $\mid \log _{2}$ (fold change) $\geq 1$ | under at least one treatment in shoots and roots, respectively (Fig. $8 \mathrm{~b}$ and Additional file 12: Table S8). As shown in Fig. 9b, 41, 27, 29 and 48 differentially expressed CsbZIP genes were identified in C. songorica shoots under heat, cold, salt and drought stress, respectively. Compared to the controls, there were 47, 41, 40 and 53 differentially expressed CsbZIP genes in roots under the heat, salt, cold and drought stress treatments, respectively (Fig. 8b and Additional file 12: Table S8). Interestingly, seven and 12 of these genes overlapped under four abiotic stress conditions in shoots and roots, respectively. A total of 77 CsbZIP genes were differentially expressed in both shoots and roots under four abiotic stress conditions. These genes were mainly distributed in subfamilies A, E and S. In subfamily E, four out of five genes were differentially expressed in shoots under abiotic stress. The heat and drought treatments had the most overlapping genes (34 in shoots; 28 in roots), whereas the cold and salt stress treatments had the fewest overlapping genes (13 in shoots, 21 in roots; Fig. 8b and Additional file 12: Table S8).

Ten stress-related CsbZIP genes were found in the co-expressed network analysis. As shown in Fig. 7c, 835 co-expressed genes were found, and some showed 


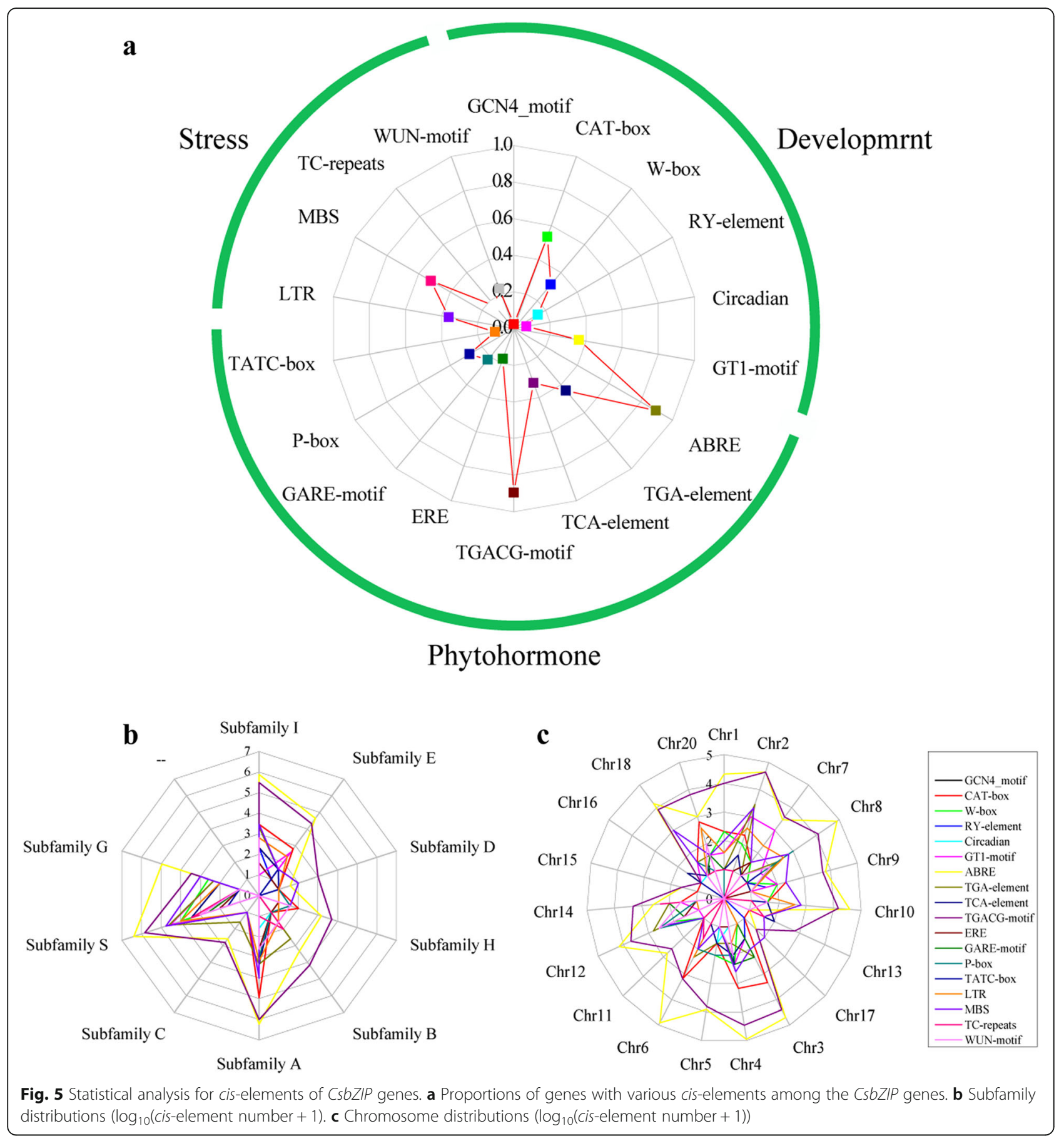

overlap with these genes. Furthermore, we analysed the GO annotation of these co-expressed genes, and some major stress-related $\mathrm{GO}$ terms were found response to stimulus (GO:0050896), response to hormone (GO: 0009725), and metabolic process (GO:0008152). Strikingly, we also found that some genes were involved in flower development, including reproductive process (GO:0022414), reproductive system development (GO: 0061458), reproductive structure development (GO:
0048608), flower development (GO:0009908), floral organ development (GO:0048437), and pollen development (GO:0009555; Additional file 13: Table S9). We further identified the ABA-dependent and ABAindependent abiotic stress-responsive CsbZIP genes. A total of 22 CsbZIP genes were differentially expressed in the ABA treatment. Among these genes, eight CsbZIP genes were classed to subfamily A. Furthermore, 22 CsbZIP genes were also responsive to both the ABA and 


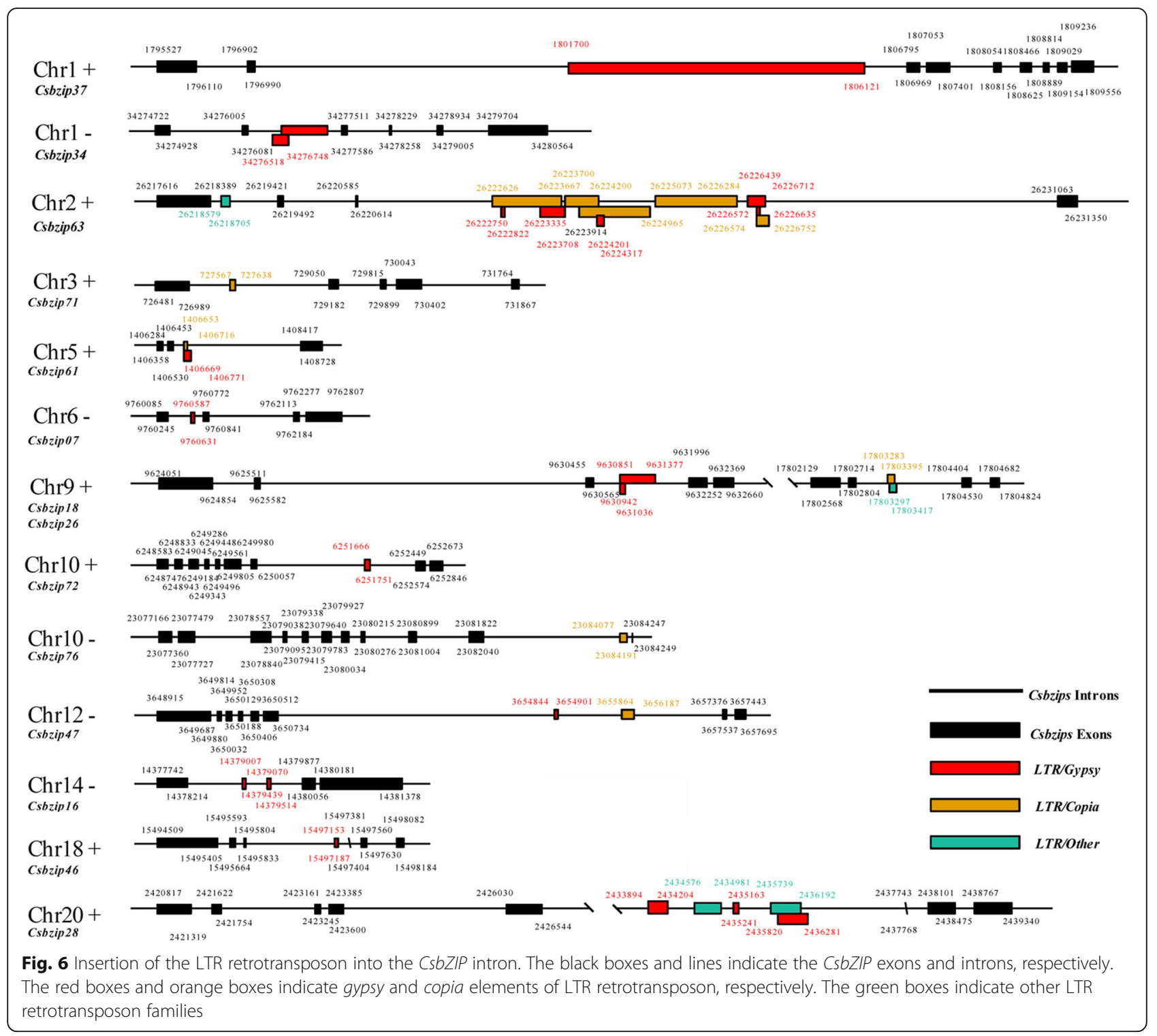

abiotic stress treatments in C. songorica, while 55 CsbZIP genes were specifically responsive to abiotic stress, indicating that more ABA-independent CsbZIP genes were involved in response to abiotic stress.

The expression patterns of some related CsbZIP genes were verified by RT-qPCR under salt stress $(100 \mathrm{mM}$ $\mathrm{NaCl} ; 24 \mathrm{~h}$ ), drought stress ( $2 \%$ soil water content), heat stress $\left(40^{\circ} \mathrm{C} ; 24 \mathrm{~h}\right)$, cold stress $\left(4{ }^{\circ} \mathrm{C} ; 24 \mathrm{~h}\right)$ and $\mathrm{ABA}$ stress $(100 \mu \mathrm{M} ; 24 \mathrm{~h})$. As shown in Fig. 9 , the result was relatively consistent with similar trends, indicating that the selected CsbZIP genes were significantly induced by these five stresses. For example, CsbZIP48, CsbZIP02 and CsbZIP33 were upregulated under heat and cold stresses. CsbZIP02 and CsbZIP22 were upregulated under drought stress.

\section{Discussion}

C. songorica is an allotetraploid plant and a major xerophyte perennial desert plant native to Northwest China that provides valuable genetic resources for understanding and improving stress resistance in plants. Currently, we have completed whole-genome sequencing and transcriptome sequencing of $C$. songorica. Many studies have shown that the bZIP family participates in different biological processes, including plant development, flowering and response to environmental stresses [35]. Although the functions of bZIP TFs are diverse, a genome survey of bZIP family genes has not yet been reported in $C$. songorica. Here, 86 bZIP family genes were found in the C. songorica genome. The distribution of the CsbZIP genes was not different in the subgenome of $C$. songorica (Additional 


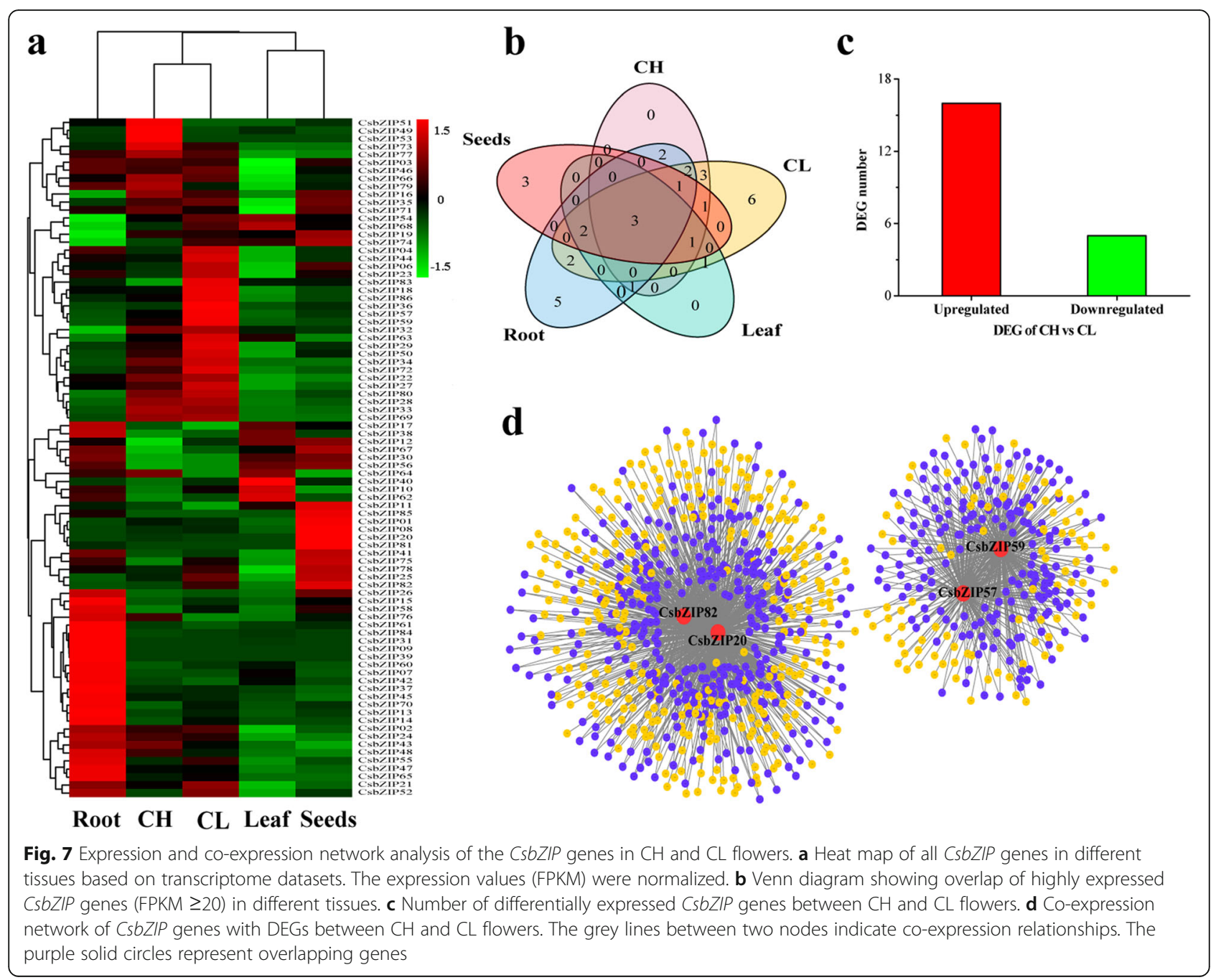

file 2: Figure S1). Compared to other plants, the result suggested that the bZIP family did not exhibit significant expansion in C. songorica. For example, 75 bZIP TFs were identified in Arabidopsis [9], 77 were found in cassava [5], and 64 were recognized in cucumber [36]. Evolutionary analysis showed that bZIP TFs from three species could be classified into 10 subfamilies by multiple sequence alignments. Strikingly, no CsbZIP genes were distributed in subfamily $\mathrm{F}$, which is involved in $\mathrm{Zn}$ transport (Fig. 1). In Arabidopsis, AtbZIP19, AtbZIP23 and AtbZIP24 from subfamily $\mathrm{F}$ were suggested to improve the plant resistance under Zn-limiting treatments [37]. These results suggested that $C$. songorica lost these functional genes during evolution.

Gene structure analysis indicated that the CsbZIP genes introns presented a large difference with numbers ranging from 0 to 10 , but there was a similar gene structure in each subfamily (Additional file 2: Figure S1). We found that approximately $24 \%$ of CsbZIP genes only had an exon and were mainly clustered in subfamily $\mathrm{S}$, with the same results as soybean and banana [21, 38]. In addition, duplicated gene pairs were also found to be classified in the same subfamily by phylogenetic analysis. For example, the segmental pairs CsbZIP32-CsbZIP68 (3 exons) and CsbZIP25-CsbZIP29 (4 exons) were classified into subfamilies $\mathrm{A}$ and $\mathrm{E}$, respectively. The CsbZIP genes of subfamilies D, C and G had more exons than all other subfamilies (Additional file 2: Figure S1). This result is supported by results from studies of soybean and Brassica napus $[27,38]$. Research has shown that the rate of intron gain by segmental duplication is slower than the rate of intron loss in rice [39]. These results might indicate that the original genes were distributed in subfamilies D, C and G. The mechanism of exon/intron gain/ loss caused diversification for the gene family [40]. Conserved motif analysis showed that all the CsbZIP proteins contained the typical bZIP domain, Furthermore, we found that motif 4 (abscisic acid-insensitive) was involved in ABA, and motif 5 was specific to subfamily A (Additional file 2: Figure S1). The DNA-binding ability 


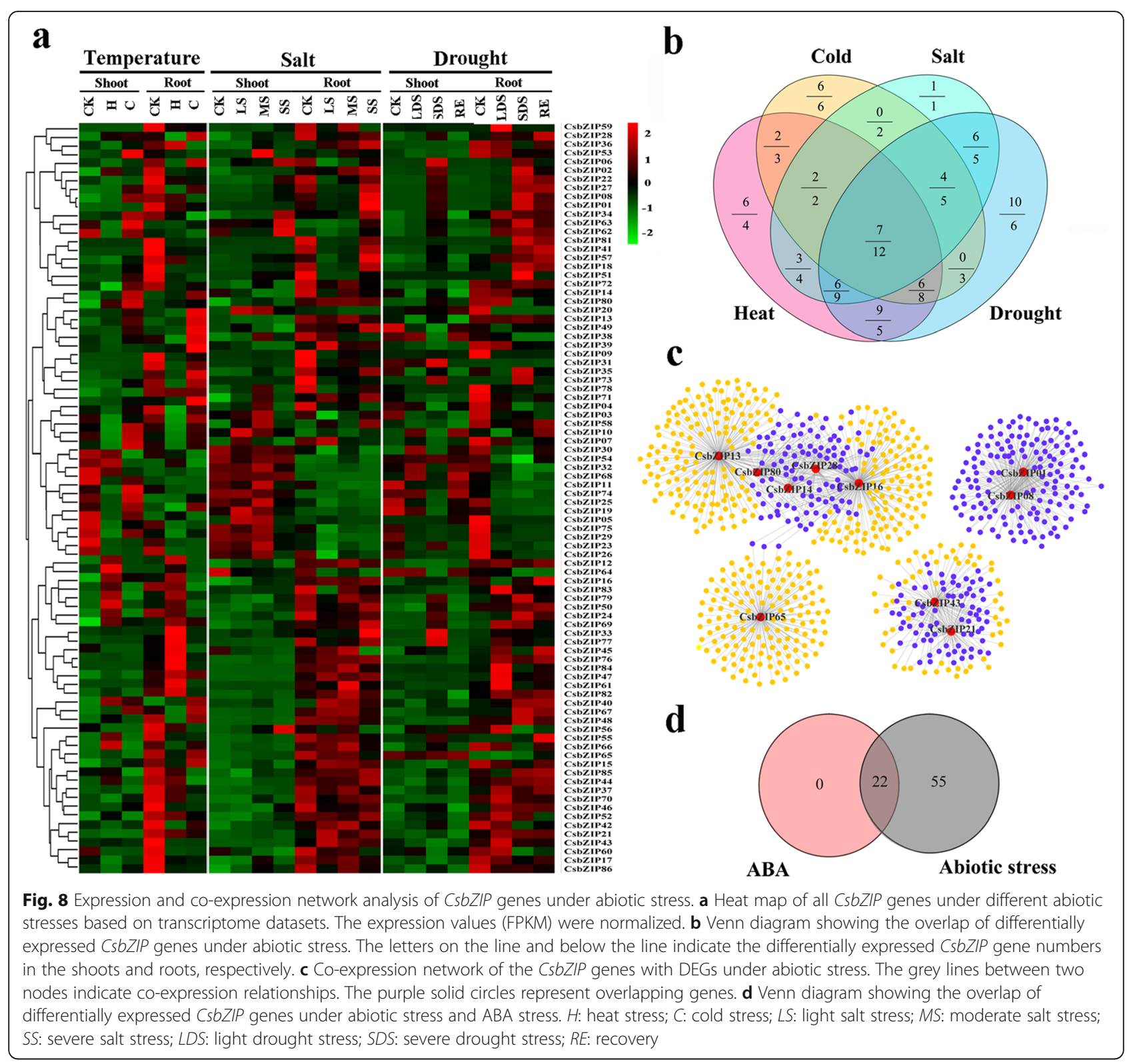

of bZIP transcription factors is depend on the basic region of the bZIP domain. Then, the specific DNAbinding is controlled by certain key amino acid residues present from the basic and hinge regions of bZIP domain. Plant bZIP transcription factors can bind to some motif ACGT core DNA sequences, including G-box, ABRE, GCN4_motif, etc. [9]. In this study, some cis-acting elements also were identified in the upstream of CsbZIP genes. In Arabidopsis, some AtbZIP genes can regulate transcription of $\mathrm{ABA}$ dependent genes that interacting with ABRE (Abscisic acid responsive element) cis-element on promoters, including $A B F 1, A B F 2$, ABF4 [41]. They belong to subfamily $\mathrm{A}$ and abscisic acid-responsive element-binding factors (ABF) [7]. In $C$. songorica, $84 \%$ CsbZIP genes contained at least one
ABRE cis-element on promoters and showed the expression change under ABA treatment in subfamily A. Furthermore, AtbZIP11 have been confirmed to modulate transcription of target gene that carry the G-box ciselement [42]. In this study, CsbZIP72 and CsbZIP76 contained the GCN_motif cis-element (G-box) that is the recognition site for bZIP transcription factors in promoters. In rice, RISBZ1, a bZIP transcription factor, are involved in storage of endosperm based on interacting with GCN4_motif [43]. These results indicated that CsbZIP72 and CsbZIP76 also participated in storage process. Together, gene structure and conserved motif analysis indicated that the relationship of CsbZIP genes in the same subfamily were closer than those in other subfamilies during the gene evolution process. 


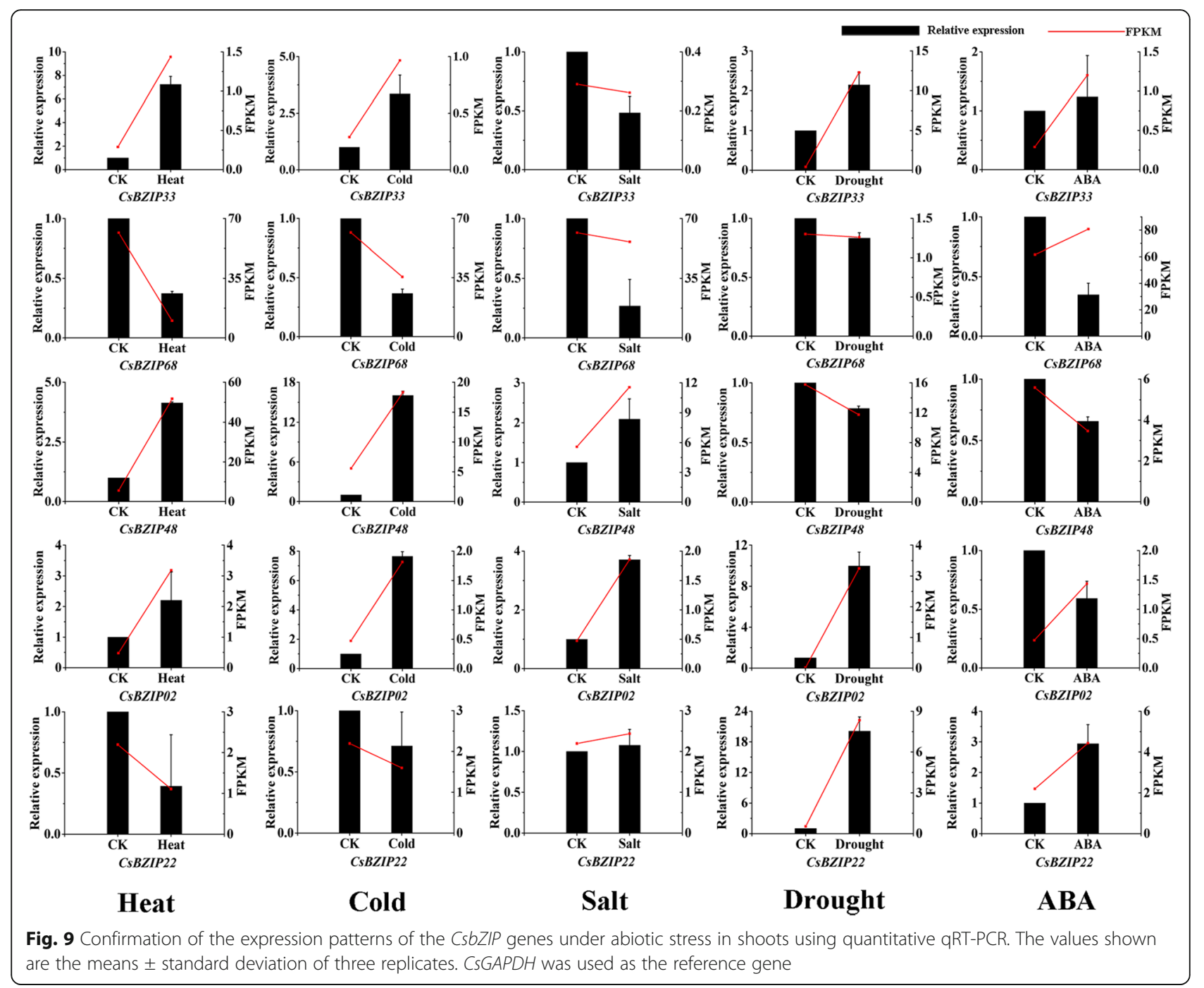

The duplication event of genes caused the generation of new genes and gene family expansion. The mechanism mainly included segmental duplication, tandem duplication, and transposition events. In this study, almost all paralogous gene pairs from C. songorica were distributed between chromosomes. In addition, 29 paralogous gene pairs $(72.5 \%)$ arose from the duplication of subgenome A and subgenome B of C. songorica (Fig. 2). In this whole-genome analysis, we also found that $C$. songorica had undergone a whole-genome duplication event in evolutionary history. These results suggested that the expansion of the C. songorica gene family was produced by whole-genome duplication events. The mechanism of comparative genomics divided the syntenic blocks by genome structures. The synteny analysis can identify the functional and evolutionary relationship between different species. We performed syntenic and phylogenetic comparisons between rice and $C$. songorica and identified orthologous gene pairs. A total of 52
OsbZIP and 73 CsbZIP genes (84.9\%) were identified as orthologs. We found 12 single orthologous gene pairs (11.7\%) between $C$. songorica and rice, indicating that these genes might be contained in the genome of the common ancestor of the two species (Fig. 3). Most gene pairs $(88.3 \%)$ showed a substantially complex relationship, including single CsbZIP genes corresponding to the multiple OsbZIP genes or single OsbZIP genes corresponding to the multiple CsbZIP genes. Furthermore, we further calculated the Ks values and $\mathrm{Ka} / \mathrm{Ks}$ ratios for the orthologous $(\mathrm{Cs}-\mathrm{Os})$ and paralogous $(C s-C s)$ gene pairs. When $\mathrm{Ka} / \mathrm{Ks}<1$, this indicates that the gene has purifying selection, but $\mathrm{Ka}$ / $\mathrm{Ks} \geq 1$ indicates positive selection for the gene $[44,45]$. The $\mathrm{Ka} / \mathrm{Ks}$ ratios of the orthologous gene pairs were lower than 0.6, indicating they might have progressed through a purifying selection during the long evolutionary history between the rice and $C$. songorica genomes (Fig. 4). 
Extensive studies have indicated the important functions of the bZIP transcription factors in regulating various stress signalling pathways. In Arabidopsis, overexpressing TabZIP6 from wheat (Triticum aestivum L.) decreased the freezing tolerance of transgenic Arabidopsis seedlings [46]. Overexpression of GhABF2, a $b Z I P$ gene from cotton (Gossypium hirsutum L.), significantly improved drought and salt stress tolerance both in transgenic Arabidopsis and cotton plants [47]. Although some stress-related bZIPs have been identified in plants, research on $C$. songorica is still lacking. Here, 79.1 and $87.2 \%$ abiotic stress-related CsbZIP genes were identified in shoots and roots, respectively. In addition, 7 and 12 genes showed differential expression under four stress conditions (Fig. 8b). These results indicated that CsbZIP genes are involved in the regulation of abiotic stresses. Gene expression analysis demonstrated that there were more stress-responsive CsbZIP genes for drought and heat than for cold and salt stress-responsive CsbZIP genes, suggesting that CsbZIP genes might have important biological functions in response to drought and high temperature. This result is supported by the fact that $C$. songorica is a native grass in desert grasslands. Furthermore, all ABA-responsive CsbZIP genes were differentially expressed under abiotic stresses. These results indicate that the CsbZIP genes participate in ABA-dependent and ABA-independent pathways.

In flowering plants, cleistogamy is a special mating system that has been found in approximately 700 species [48-50], such as Pseudostellaria heterophylla and Viola philippica [51, 52]. The types of cleistogamy can be divided into dimorphic cleistogamy, complete cleistogamy and induced cleistogamy [50]. Cleistogamy is suspected to be beneficial to plants because CL flowers can ensure seed production by selfpollination under extreme environmental conditions [53]. C. songorica, which undergoes dimorphic cleistogamy, produces both closed (cleistogamous) flowers and open (chasmogamous) flowers on the same individual, but the flowers appear in different positions. Unlike $\mathrm{CH}$ flower production at the apical meristem, the CL flower is produced in the leaf sheath and plays a crucial role in seed yield in C. songorica [30]. In this study, 21 CsbZIPs were differentially expressed between $\mathrm{CH}$ and $\mathrm{CL}$ flowers, including 2 FD genes (Fig. 7c). In Arabidopsis, FT genes were found to regulate the flowering time and floral induction at the meristem [10]. APETALA1 (AP1) is target genes for $F T$ genes and belong to the classic ABCE model. AP1 is involved in floral meristem specification and perianth identity $[54,55]$. In $P$. heterophylla, the A class gene $A P 1$ is highly expressed in CL flowers [51]. Furthermore, $F D$, a bZIP TFs, was found to play a core role in the $F T$ activation of floral identity genes such as AP1 in Arabidopsis [10]. In our study, two FD genes were differentially expressed between $\mathrm{CH}$ and $\mathrm{CL}$ flowers in C. songorica (CsbZIP58 and CsbZIP60). Strikingly, CsbZIP58 and CsbZIP60 were paralogous gene pairs and were orthologous genes for OsbZIP77, which is named OsFD1. OsFD1 functions in the activation of the AP1/FUL genes and promotes flowering [56].

The WGCNA analysis showed that some stressresponsive CsbZIP genes were co-expressed with genes involved in flower development. Additionally, some CL flower-related CsbZIP genes were also co-expressed with genes that responded to stress (Fig. $7 d$ and Fig. $8 \mathrm{c}$ ). We further found that 17 common CsbZIP genes showed differential expression under stress response and during $\mathrm{CL}$ flowering, revealing that these genes may involve numerous developmental processes in C. songorica (Fig. 10). Four CsbZIP genes were selected to verify the expression pattern in CL flowers and $\mathrm{CH}$ flowers and under abiotic stress by RT-qPCR. The expression patterns of selected genes showed significant expression changes between the $\mathrm{CH}$ and $\mathrm{CL}$ flowers and floral primordium (CHP, CLP). Strikingly, these genes also showed significant expression changes and were barely downregulated under abiotic stress. For example, CsbZIP26, CsbZIP75 and CsbZIP60 were differentially expressed under drought stress. CsbZIP60 and CsbZIP58 were differentially expressed under heat stress (Fig. 10). In Cardamine kokaiensis, 69 differentially expressed genes related to floral development and cold stress were identified in $\mathrm{CH}$ and CL flowers [57]. Furthermore, the study of $P$. heterophylla also indicated that significantly differentially expressed between $\mathrm{CH}$ and $\mathrm{CL}$ flowers were involved in defence responses [51]. These results may show that CL flowers might be affected by environmental stresses.

\section{Conclusion}

In summary, this study represents the first comprehensive identification and analysis of the bZIP TF family in $C$. songorica. CL flower development and stress response CsbZIP genes were highlighted for their expression patterns and expression networks. These results may provide useful insights into the CsbZIP genes in the stress response and CL flower development in future studies.

\section{Methods}

\section{Identification of the $b Z I P$ genes in $C$. songorica}

The bZIP protein and gene sequences from rice (Oryza sativa) and Arabidopsis were acquired from Phytozome12 (https://phytozome.jgi.doe.gov/pz/portal.html). BLAST searches were used to identify the candidate bZIPs in the $C$. songorica genome (not published data) with rice and Arabidopsis bZIP sequences as a query $(e-$ value cut-off $\left.>1 \mathrm{e}^{-5}\right)$. Then, conserved domain and 


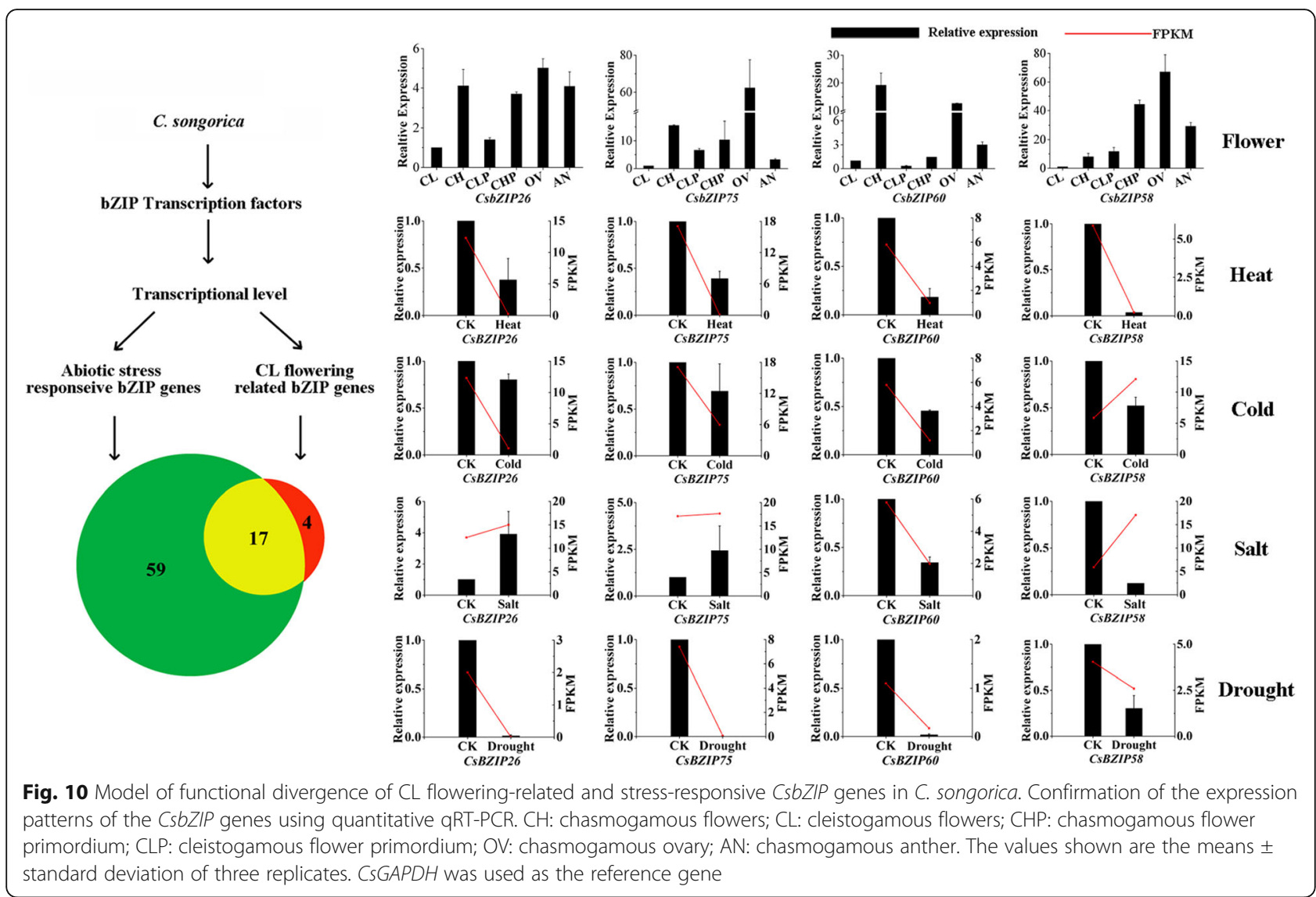

redundant sequence of all possible protein sequences were further examined the with PFAM (https://pfam. sanger.ac.uk/; $e$-value cut-off $\left.>1 \mathrm{e}^{-5} ; \mathrm{PF} 00170\right)$ and $\mathrm{CD}$ HIT (http://weizhongli-lab.org/cdhit_suite/cgi-bin/index. cgi) tools, respectively.

\section{Protein properties, phylogenetic classification, gene} structure and conserved motif analysis

The CsbZIP amino acid residues, grand average of hydropathicity, isoelectric points and molecular weights were analysed by ExPaSy (http://web.expasy. org/protparam/) [58]. The online tool GSDS was used to show the gene structure of CsbZIP genes (http:// gsds.cbi.pku.edu.cn/). The conserved motif was predicted by the MEME program with a maximum number of motifs 20 (between 10 to 50 optimum width; http://meme-suite.org/) [59]. Then, MEGA 7 software was used to construct the phylogenetic tree of three species by the neighbour-joining method (1000 bootstrap replicates, http://www.megasoftware.net). WoLF PSORT (https://wolfpsort.hgc.jp/) were used to predict the subcellular locations of $C$. songorica bZIP proteins.

\section{Synteny analysis and chromosome localization}

The following three steps were used to identify the paralogous and orthologous gene pairs, 1) OrthoMCL software V5 was used with default settings [60]; 2) BLASTP was used to multiple sequence alignment $\left(\mathrm{E}<1 \mathrm{e}^{-5}\right.$, top 3 matches) within the $C$. songorica genome and between the $C$. songorica and rice genomes; 3) MCL software was used to cluster pairs in to OrthoMCL groups (values for finding clustering of different granularity $=1$ ). The Circos (Circos 0.69) program was used to show the gene pairs [61]. The synonymous (Ks) and non-synonymous (Ka) nucleotide substitutions between paralogous and orthologous gene pairs were calculated based on the comparative synteny map of the $C$. songorica genome and between the rice and $C$. songorica genomes, using Clustal W, PAL2NAL [62] and the yn00 program of the biopipeline (https://github.com/tanghaibao/bio-pipeline/ tree/master/synteny-pipeline).

\section{Identification of putative cis-elements and LTR retrotransposon in CsbZIP genes}

The $1500 \mathrm{bp}$ upstream sequences of the CsbZIP genes were used to identify the cis-elements in the promoter 
regions with the PlantCARE [63] website (http://bioinformatics.psb.ugent.be/webtools/plantcare/html/).

LTR-FINDER (1.0.5 version) was used to identify the LTR retrotransposon in CsbZIP genes (http://tlife.fudan. edu.cn/ltr_finder/) [64].

\section{Stress treatment and transcriptomic data analysis}

Four-week-old seedlings of $C$. songorica were grown in a growth chamber that was maintained at $16 \mathrm{~h}$ light $28^{\circ} \mathrm{C} /$ $8 \mathrm{~h}$ dark $24{ }^{\circ} \mathrm{C}$, with an irradiance of $150 \mu \mathrm{mol}$ quanta $\mathrm{m}^{-2} \mathrm{~s}^{-1}$ and $65 \%$ relative humidity.

Each pot was filled with a sand/vermiculite $(1 / 1, \mathrm{v} / \mathrm{v})$ mixture, with $0.45 \mathrm{~kg}$ per pot. Each plant was irrigated with $100 \mathrm{~mL}$ Hoagland nutrient solution every 3 days. The 9-week-old seedlings were treated with $40^{\circ} \mathrm{C}$ (heat), $4{ }^{\circ} \mathrm{C}$ (cold), $50 \mathrm{mM} \mathrm{NaCl}$ (light salt stress), $100 \mathrm{mM} \mathrm{NaCl}$ (moderate salt stress), $200 \mathrm{mM} \mathrm{NaCl}$ (severe salt stress) and $100 \mu \mathrm{M}$ ABA (ABA stress). The shoots and roots of each plant were collected $24 \mathrm{~h}$ after treatment application in the growth medium, immediately frozen in liquid nitrogen, and stored at $-80^{\circ} \mathrm{C}$. Transcriptome data of CsbZIP genes under drought stress were obtained from our previous study [65].

Total RNA of C. songorica shoot and root under abiotic stresses were extracted with TRIzol reagent (Invitrogen, USA). The library preparation and deep sequencing were performed by the Novogene Bioinformatics Technology Cooperation (Beijing, China). Sequencing libraries were generated using NEBNext ${ }^{\circ}$ Ultra $^{\mathrm{am}}$ RNA Library Prep Kit for Illumina ${ }^{\oplus}$ (NEB, USA) according manufacturer's recommendations. The libraries were then sequenced on a HiSeq2500 with a sequencing read length of $125 \mathrm{bp}$. Clean reads were obtained by removing reads containing adapters, reads containing poly- $\mathrm{N}$ and lower quality reads from the raw reads. The clean reads were mapped to the C. songorica genome (data not published) using HISAT2 from the BMK Cloud server (www.biocloud.net). Quantification of gene expression levels were estimated by fragments per kilobase of transcript per million fragments (FPKM) mapped using StringTie (1.3.1) in each sample $[66,67]$. DESeq $R$ package (1.10.1) provide statistical routines for determining differential expression in digital gene expression data using a model based on the negative binomial distribution. The resulting FDR (false discovery rate) were adjusted using the PPDE (posterior probability of being DE). Genes with an adjusted FDR $<0.01$ and $\mid \log 2$ (foldchange) $\geq 1$ found by DESeq were assigned as differentially expressed.

Finally, a heat map of the CsbZIP genes expression profile was shown by the OmicShare tools, a free online platform for data analysis (http://www.omicshare.com/ tools). A Venn diagram was generated by the jvenn website (http://jvenn.toulouse.inra.fr/app/example.html).
Expression profiles of CsbZIP genes in different tissues of C. songorica

Transcription analysis were performed to identify expression patterns in different tissues, including leaves, roots, seeds, chasmogamous $(\mathrm{CH})$ flowers and cleistogamous (CL) flowers. Roots and leaves samples were obtained from 6-week-old C. songorica seedings. Seed samples were mixed with imbibed seeds and germinated seeds. The samples of $\mathrm{CH}$ flowers, $\mathrm{CL}$ flowers were collected at the green anther stage of $C$. songorica. The methods of RNA-seq and data analysis were the same as described previously.

\section{Co-expression network construction and enrichment analysis}

The co-expression network analysis of CsbZIP genes involved in CL flower development and stress response were performed with weighted gene co-expression network (WGCNA; https://horvath.genetics.ucla.edu/html/CoexpressionNetwork/Rpackages/WGCNA/Tutorials/) analysis. For abiotic stress analysis, 59 RNA-seq data were used for WGCNA analysis. For CL flower development, 14 RNA-seq data were used for WGCNA analysis, including CL flowers and $\mathrm{CH}$ flowers. The co-expression network was shown by Cytoscape (v 3.5.1; https://cytoscape.org/) software. The coexpression genes were annotated using KOBAS 3.0 (http:// kobas.cbi.pku.edu.cn/download.php).

\section{Quantitative real-time (RT) PCR}

Total RNA was isolated from C. songorica shoots after stress treatments for qRT-PCR using RNAiso reagent (TaKaRa, Dalian, China). qRT-PCR was performed using SYBR Premix Ex TaqTM (TaKaRa). Approximately $1 \mu \mathrm{g}$ of RNA was reverse-transcribed into first-stand cDNA with the PrimeScript ${ }^{\circ}$ RT reagent Kit (TaKaRa), and the product was used as a template for qRT-PCR with specific primers (Additional file 14: Table S10). CsGAPDH was used as the reference gene. The relative expression levels were calculated by the comparative CT method. All reactions were performed in triplicate.

\section{Supplementary information}

Supplementary information accompanies this paper at https://doi.org/10. 1186/s12864-019-6092-4.

Additional file 1: Table S1. Basic characteristic of CsbZIP genes in C. songorica.

Additional file 2: Figure S1. Gene structure and conserved motif of CsbZIP genes based on the evolutionary relationship. a Gene structure analyses were presented with GSDS. The blue boxes indicate upstream / downstream, the orange boxes represent exons, and the black lines indicate introns. b All motifs were identified by MEME database with the complete amino acid sequences of CsbZIPs. Each motif was showed by different colored block, with their numbers in the center of the motifs. The number in boxes (1-20) represents motif 1 - motif 20, respectively. 
The position and length of each colored box represents the actual motif size. The evolutionary tree was carried out with MEGA7.

Additional file 3: Figure S2. The major motifs identified by MEME in the putative CsbZIP proteins.

Additional file 4: Figure S3. Gene location of bZIP genes in C. songorica. a Statistical analysis of amino acid residues, grand average of hydropathicity, isoelectric points and molecular weight of bZIP genes in C. songorica. b The distribution of bZIP genes on C. songorica chromosomes, shown as percentages.

Additional file 5: Table S2. Synteny blocks of bZIP genes within C. songorica genome.

Additional file 6: Table S3. Synteny blocks of bZIP genes between $C$. songorica and rice genome.

Additional file 7: Table S4. Cis-elememts analysis of CsbZIP genes. Additional file 8: Table S5. Expression pattern of CsbZIP genes in different organ.

Additional file 9: Table S6. Go enrichment of DEG between $\mathrm{CH}$ and $\mathrm{CL}$ flowers.

Additional file 10 Table S7. Gene list and Go annotation of co-expression genes with selected CsbZIP gene involved in CL flower development.

Additional file 11: Figure S4. GO enrichment analysis of co-expression genes with CsbZIP20, CsbZIP57, CsbZIP59, CsbZIP82.

Additional file 12: Table S8. Transcriptome data of CsbZIP genes in shoot and root under abiotic stress.

Additional file 13: Table S9. Gene list and $G O$ enrichment analysis of co-expression genes with abiotic related genes.

Additional file 14: Table S10. Primer list for gene specific primers.

\section{Abbreviations}

bZIP: Basic leucine zipper; CH: Chasmogamous; CL: Cleistogamous; Ka: The rate of nonsynonymous substitutions; Ks: The rate of synonymous substitutions; qRT-PCR: Quantitative real-time reverse transcription PCR; TFs: Transcription factors; WGCNA: Weighted gene co-expression network

\section{Acknowledgements}

We would like to thank the reviewers for their helpful comments on the original manuscript.

\section{Authors' contributions}

QY concevied and designed the experments, carried out the boinformatics analyses, and drafted the manuscript. FW, QM and JL particpated in the data analysis. XFZ, TTM and YFZ helped to handle figure an tables. YRW and JYZ conceived and directed the study, parricipated in its design and helped to draft the manscript. JYZ, as the correspondebce author, provided fincial supprot for the article and designed the expermets. All authors read and approved the final manuscript.

\section{Funding}

National Natural Science Foundation of China (31572453), Program for Changjiang Scholars and Innovative Research Team in University (IRT_17R50), Fundamental Research Funds for the Central Universities (Izujbky-2016-10), and the Open Project Program of Arid Meteorology (IAM201703). All these funding play roles in the design of the study and collection, analysis, and in writing the manuscript.

\section{Availability of data and materials}

The datasets generated and analysed during the current study are not publicly available due to the whole genome sequencing work of $C$. songorica has not been published but are available from the corresponding author on reasonable request.

Ethics approval and consent to participate

C. songorica, a cultivar, was used in this research. This cultivar was obtained by domesticating wild plants, cultivating by College of Pastoral Agriculture Science and Technology, Lanzhou University.
Consent for publication

Not applicable.

\section{Competing interests}

The authors declare that they have no competing interests.

Received: 1 April 2019 Accepted: 10 September 2019

Published online: 22 October 2019

\section{References}

1. Nijhawan A, Jain M, Tyagi AK, Khurana JP. Genomic survey and gene expression analysis of the basic leucine zipper transcription factor family in rice. Plant Physiol. 2008;146(2):333-50

2. Talanian RV, Mcknight CJ, Kim PS. Sequence-specific DNA binding by a short peptide dimer. Science. 1990;249(4970):769-71.

3. Hurst HC. Transcription factors 1: bZIP proteins. Protein Profile. 1995:2(2):123-68.

4. Liu X, Chu Z. Genome-wide evolutionary characterization and analysis of bZIP transcription factors and their expression profiles in response to multiple abiotic stresses in Brachypodium distachyon. BMC Genomics. 2015;16:227.

5. Hu W, Yang H, Yan Y, Wei Y, Tie W, Ding Z, Zuo J, Peng M, Li K. Genomewide characterization and analysis of bZIP transcription factor gene family related to abiotic stress in cassava. Sci Rep. 2016;6:22783.

6. Glover JN, Harrison SC. Crystal structure of the heterodimeric bZIP transcription factor C-Fos-C-Jun bound to DNA. Nature. 1995;373(6511):257.

7. Yoshida T, Fujita Y, Sayama H, Kidokoro S, Maruyama K, Mizoi J, Shinozaki K, Yamaguchishinozaki K. AREB1, AREB2, and ABF3 are master transcription factors that cooperatively regulate ABRE-dependent ABA signaling involved in drought stress tolerance and require ABA for full activation. Plant J. 2010; 61(4):672-85.

8. Fujita Y, Yoshida T, Yamaguchi-Shinozaki K. Pivotal role of the AREB/ABFSnRK2 pathway in ABRE-mediated transcription in response to osmotic stress in plants. Physiol Plant. 2013;147(1):15-27.

9. Jakoby M, Weisshaar B, Drögelaser W, Vicentecarbajosa J, Tiedemann J, Kroj T, Parcy F. bZIP transcription factors in Arabidopsis. Trends Plant Sci. 2002; 7(3):106-11.

10. Abe M, Kobayashi $Y$, Yamamoto S, Daimon $Y$, Yamaguchi A, Ikeda $Y$, Ichinoki $H$, Notaguchi M, Goto K, Araki T. FD, a bZIP protein mediating signals from the floral pathway integrator FT at the shoot apex. Science. 2005;309(5737):1052-6.

11. Kwon S, Kang NK, Koh HG, Shin SE, Lee B, Jeong BR, Chang YK. Enhancement of biomass and lipid productivity by overexpression of a bZIP transcription factor in Nannochloropsis salina. Biotechnol Bioeng. 2017;115:10.

12. Dash M, Yordanov YS, Georgieva T, Tschaplinski TJ, Yordanova E, Busov V. Poplar PtabZIP1-like enhances lateral root formation and biomass growth under drought stress. Plant J Cell Mol Biol. 2017:89(4):692

13. Iven T, Strathmann A, Böttner S, Zwafink T, Heinekamp T, Guivarc'H A, Roitsch T, Drögelaser W. Homo- and heterodimers of tobacco bZIP proteins counteract as positive or negative regulators of transcription during pollen development. Plant J. 2010;63(1):155-66.

14. Wei K, Chen J, Wang Y, Chen Y, Chen S, Lin Y, Pan S, Zhong X, Xie D. Genome-wide analysis of bZIP-encoding genes in maize. Dna Res. 2012; 19(6):463-76.

15. Jang S, Li HY, Kuo ML. Ectopic expression of Arabidopsis FD and FD PARALOGUE in rice results in dwarfism with size reduction of spikelets. Sci Rep. 2017;7:44477

16. Gibalová A, Reňák D, Matczuk K, Dupl'Áková N, Cháb D, Twell D, Honys D. AtbZIP34 is required for Arabidopsis pollen wall patterning and the control of several metabolic pathways in developing pollen. Plant Mol Biol. 2009; 70(5):581

17. Zong W, Tang N, Yang J, Peng L, Ma S, Xu Y, Li G, Xiong L. Feedback regulation of $A B A$ signaling and biosynthesis by a bZIP transcription factor targets drought resistance related genes. Plant Physiol. 2016;171(4):2810.

18. Huang C, Zhou J, Jie Y, Xing H, Zhong Y, She W, Wei G, Yu W, Ma Y. A ramie (Boehmeria nivea) bZIP transcription factor BnbZIP3 positively regulates drought, salinity and heavy metal tolerance. Mol Breed. 2016:36(8):120.

19. Wang J, Li Q, Mao X, Li A, Jing R. Wheat transcription factor TaAREB3 participates in drought and freezing tolerances in Arabidopsis. Int J Biol Sci. 2016;12(2):257.

20. J F TS, S I IY, Y K YT. Repression of shoot growth, a bZIP transcriptional activator, regulatescell elongation by controlling the level of gibberellins. Plant Cell. 2000;12(6):901-15. 
21. He S, Shan W, Kuang JF, Xie H, Xiao YY, Lu WJ, Chen JY. Molecular characterization of a stress-response bZIP transcription factor in banana. Plant Cell Tissue Org Cult. 2013;113(2):173-87.

22. Tu M, Wang X, Huang L, Guo R, Zhang H, Cai J, Wang X. Expression of a grape bZIP transcription factor, VqbZIP39, in transgenic Arabidopsis thaliana confers tolerance of multiple abiotic stresses. Plant Cell Tissue Org Cult. 2016;125(3):537-51.

23. Lee SC, Choi HW, Hwang IS, Du SC, Hwang BK. Functional roles of the pepper pathogen-induced bZIP transcription factor, CAbZIP1, in enhanced resistance to pathogen infection and environmental stresses. Planta. 2006; 224(5):1209-25.

24. Li X, Fan S, Hu W, Liu G, Wei Y, He C, Shi H. Two cassava basic leucine zipper (bZIP) transcription factors (MebZIP3 and MebZIP5) confer disease resistance against cassava bacterial blight. Front Plant Sci. 2017:8(2110):2110.

25. Zhu M, Meng X, Cai J, Li G, Dong T, Li Z. Basic leucine zipper transcription factor SIbZIP1 mediates salt and drought stress tolerance in tomato. BMC Plant Biol. 2018;18(1):83.

26. Liu C, Mao B, Ou S, Wang W, Liu L, Wu Y, Chu C, Wang X. OsbZIP71, a bZIP transcription factor, confers salinity and drought tolerance in rice. Plant Mol Biol. 2014;84(1-2):19.

27. Wang P, Yang C, Chen H, Luo L, Leng Q, Li S, Han Z, Li X, Song C, Zhang X, et al. Exploring transcription factors reveals crucial members and regulatory networks involved in different abiotic stresses in Brassica napus L. BMC Plant Biol. 2018;18(1):202.

28. Wang XL, Chen X, Yang TB, Cheng Q, Cheng ZM. Genome-wide identification of bZIP family genes involved in drought and heat stresses in strawberry (Fragaria vesca). Int J Genomics. 2017;2017(4860):3981031.

29. Yang J, Zhu G, Gao G, Wei Z, Han G. Effects of grazing systems on the reproductive feature of key plant population in Stipa breviflora steppe. J Arid Land Resourc Environ. 2001; 5(15):112-6.

30. Wu F, Zhang D, Muvunyi BP, Yan Q, Zhang Y, Yan Z, Cao M, Wang Y, Zhang J. Analysis of microRNA reveals cleistogamous and chasmogamous floret divergence in dimorphic plant. Sci Rep. 2018;8(1):6287.

31. Zhang J, Duan Z, Zhang D, Zhang J, Di H, Wu F, Wang Y. Co-transforming bar and CSLEA enhanced tolerance to drought and salt stress in transgenic alfalfa (Medicago sativa L.). Biochem Biophys Res Commun. 2016;472(1):75-82.

32. Zhang J, Kong L, Liu Z, Jahufer Z, Duan Z, Huo Y, Di H, Wang Y. Stress-induced expression in Arabidopsis with a Dehydrin LEA protein from Cleistogenes songorica, a xerophytic desert grass. Plant Omics. 2015;8(6):485-92.

33. Zhang J, Zhen D, Jahufer Z, An SJ, Wang YR. Stress-inducible expression of a Cleistogenes songorica ALDH gene enhanced drought tolerance in transgenic Arabidopsis thaliana. Plant Omics. 2014;7(6):438-44.

34. Duan Z, Zhang D, Zhang J, Di H, Wu F, Hu X, Meng X, Luo K, Zhang J, Wang Y. Co-transforming bar and CSALDH genes enhanced resistance to herbicide and drought and salt stress in transgenic alfalfa (Medicago sativa L.). Front Plant Sci. 2015;6:1115

35. Wolfgang DL, Snoek BL, Berend S, C hristoph W. The Arabidopsis bZIP transcription factor family-an update. Curr Opin Plant Biol. 2018;45(Pt A:36-49.

36. Baloglu MC, Eldem V, Hajyzadeh M, Unver T. Genome-wide analysis of the bZIP transcription factors in cucumber. PLoS One. 2014;9(4):e96014.

37. Assunção AGL, Koornneef M. The Arabidopsis thaliana transcription factors bZIP19 and bZIP23 regulate the adaptation to zinc deficiency. Proc Natl Acad Sci U S A. 2010;107(22):10296-301.

38. Zhang M, Liu Y, Shi H, Guo M, Chai M, He Q, Yan M, Cao D, Zhao L, Cai H. Evolutionary and expression analyses of soybean basic leucine zipper transcription factor family. BMC Genomics. 2018;19(1):159.

39. Lin H, Zhu W, Silva JC, Gu X, Buell CR. Intron gain and loss in segmentally duplicated genes in rice. Genome Biol. 2006;7(5):R41.

40. Wang L, Yin X, Cheng C, Wang H, Guo R, Xu X, Zhao J, Zheng Y, Wang X. Evolutionary and expression analysis of a MADS-box gene superfamily involved in ovule development of seeded and seedless grapevines. Mol Genet Genomics. 2015;290(3):825-46.

41. Fujita Y, Fujita AM, Satoh CR, Maruyama CK, Parvez AMM, Seki AMJPC. AREB1 is a transcription activator of novel ABRE-dependent ABA signaling that enhances drought stress tolerance in Arabidopsis. Plant Cell. 2005;17(12):3470.

42. Rook F, Gerrits N, Kortstee A, Kampen MV, Borrias M, Weisbeek P, Smeekens SJPJ. Sucrose-specific signalling represses translation of the Arabidopsis ATB2 bZIP transcription factor gene. Plant J. 2010;15(2):253-63.

43. Woo YJ. Regulatory targeting genes of RISBZ1, a seed specific transcription factor, with protein binding microarray. International Plant \& Animal Genome Conference XXl; 2013.
44. Cannon SB, Mitra A, Baumgarten A, Young ND, May G. The roles of segmental and tandem gene duplication in the evolution of large gene families in Arabidopsis thaliana. BMC Plant Biol. 2004;4:1):1-21.

45. Shiu SH, Karlowski WM, Pan R, Tzeng YH, Mayer KF, Li WH. Comparative analysis of the receptor-like kinase family in Arabidopsis and rice. Plant Cell. 2004;16(5):1220-34.

46. Cai W, Yang Y, Wang W, Guo G, Liu W, Bi C. Overexpression of a wheat (Triticum aestivum L.) bZIP transcription factor gene, TabZIP6, decreased the freezing tolerance of transgenic Arabidopsis seedlings by down-regulating the expression of CBFs. Plant Physiol Biochem. 2018;124:100-11.

47. Liang C, Meng Z, Meng Z, Malik W, Yan R, Lwin KM, Lin F, Wang Y, Sun G, Zhou T. GhABF2, a bZIP transcription factor, confers drought and salinity tolerance in cotton (Gossypium hirsutum L.). Sci Rep. 2016;6:35040.

48. Lord EM. Cleistogamy: a tool for the study of floral morphogenesis, function and evolution. Bot Rev. 1981:47(4):421-49.

49. Plitmann U. Distribution of dimorphic flowers as related to other elements of the reproductive strategy. Plant Species Biol. 2010;10(1):53-60.

50. Culley TM, Klooster MR. The cleistogamous breeding system: a review of its frequency, evolution, and ecology in angiosperms. Bot Rev. 2007;73(1):1

51. Yan L, Hu JY, Lu L, Luo YL, Wang PF, Song BH. Genome-wide analysis of gene expression reveals gene regulatory networks that regulate chasmogamous and cleistogamous flowering in Pseudostellaria heterophylla (Caryophyllaceae). BMC Genomics. 2016;17(1):382.

52. Li Q, Huo Q, Wang J, Zhao J, Sun K, He C. Expression of B-class MADS-box genes in response to variations in photoperiod is associated with chasmogamous and cleistogamous flower development in Viola philippica. BMC Plant Biol. 2016;16(1):151.

53. Darwin C. The different forms of flowers on plants of the same species: Introduction. W Pickering; 2010.

54. Irish VF, Sussex IM. Function of the apetala-1 gene during Arabidopsis floral development. Plant Cell. 1990;2(8):741-53.

55. Mandel MA, Gustafsonbrown C, Savidge B, Yanofsky MF. Molecular characterization of the Arabidopsis floral homeotic gene APETALA1. Nature. 1992;360(6401):273-7.

56. Taoka K, Ohki I, Tsuji H, Furuita K, Hayashi K, Yanase T, Yamaguchi M, Nakashima C, Purwestri YA, Tamaki S, et al. 14-3-3 proteins act as intracellular receptors for rice Hd3a florigen. Nature. 2011;476(7360):332-5.

57. Morinaga SI, Nagano AJ, Miyazaki S, Kubo M, Demura T, Fukuda H, Sakai S, Hasebe M. Ecogenomics of cleistogamous and chasmogamous flowering: genome-wide gene expression patterns from cross-species microarray analysis in Cardamine kokaiensis (Brassicaceae). J Ecol. 2008;96(5):1086-97.

58. Gasteiger E, Gattiker A, Hoogland C, Ivanyi I, Appel RD, Bairoch A. ExPASy: the proteomics server for in-depth protein knowledge and analysis. Nucleic Acids Res. 2003:31(13):3784-8.

59. Paul B, Laura B, Richard H, Jim B, Jonathan DM, Sascha O. MEME-LaB: motif analysis in clusters. Bioinformatics. 2013;29(13):1696-7.

60. Li, Research LJG. OrthoMCL: identification of ortholog groups for eukaryotic genomes. Genome Res. 2003;13(9):2178-89.

61. Krzywinski M, Schein Jl. Circos: an information aesthetic for comparative genomics. Genome Res. 2009;19(9):1639-45.

62. Mikita S, David T, Peer B. PAL2NAL: robust conversion of protein sequence alignments into the corresponding codon alignments. Nucleic Acids Res. 2006:34:W609.

63. Lescot M, Dehais $P$, Thijs $G$, Marchal $K$, Moreau $Y$, Van de Peer $Y$, Rouze $P$, Rombauts S. PlantCARE, a database of plant cis-acting regulatory elements and a portal to tools for in silico analysis of promoter sequences. Nucleic Acids Res. 2002;30(1):325-7.

64. Zhao X, Hao W. LTR_FINDER: an efficient tool for the prediction of fulllength LTR retrotransposons. Nucleic Acids Res. 2007;35:W265-8.

65. Yan Q, Wu F, Yan Z, Li J, Ma T, Zhang Y, Zhao Y, Wang Y, Zhang J. Differential co-expression networks of long non-coding RNAs and mRNAs in Cleistogenes songorica under water stress and during recovery. BMC Plant Biol. 2019;19(1):23.

66. Kim D, Langmead B, Salzberg SL. HISAT: a fast spliced aligner with low memory requirements. Nat Methods. 2015;12(4):357-60.

67. Pertea M, Pertea GM, Antonescu CM, Chang TC, Mendell JT, Salzberg SL. StringTie enables improved reconstruction of a transcriptome from RNA-seq reads. Nat Biotechnol. 2015;33(3):290-5.

\section{Publisher's Note}

Springer Nature remains neutral with regard to jurisdictional claims in published maps and institutional affiliations. 